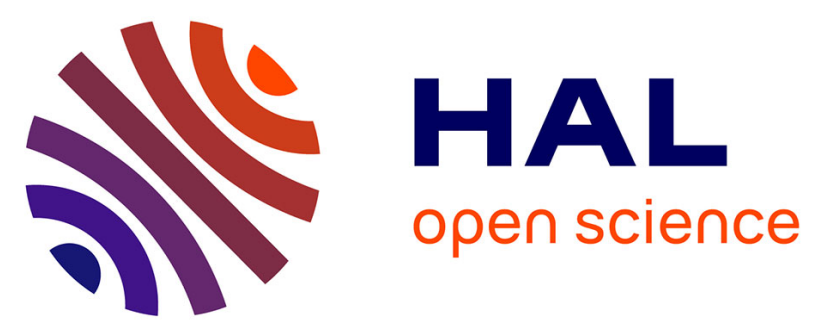

\title{
Leishmania DNA is rapidly degraded following parasite death: an analysis by microscopy and real-time PCR.
} Eric Prina, Emeric Roux, Denise Mattei, Geneviève Milon

\section{To cite this version:}

Eric Prina, Emeric Roux, Denise Mattei, Geneviève Milon. Leishmania DNA is rapidly degraded following parasite death: an analysis by microscopy and real-time PCR.. Microbes and Infection, 2007, 9 (11), pp.1307-15. 10.1016/j.micinf.2007.06.005 . pasteur-00181350

HAL Id: pasteur-00181350

https://hal-pasteur.archives-ouvertes.fr/pasteur-00181350

Submitted on 5 Nov 2007

HAL is a multi-disciplinary open access archive for the deposit and dissemination of scientific research documents, whether they are published or not. The documents may come from teaching and research institutions in France or abroad, or from public or private research centers.
L'archive ouverte pluridisciplinaire HAL, est destinée au dépôt et à la diffusion de documents scientifiques de niveau recherche, publiés ou non, émanant des établissements d'enseignement et de recherche français ou étrangers, des laboratoires publics ou privés. 


\title{
Leishmania DNA is rapidly degraded following parasite death: An analysis by microscopy and real-time PCR.
}

\author{
Eric Prina $^{\mathrm{a}, *}$, Emeric Roux ${ }^{\mathrm{b}}$, Denise Mattei ${ }^{\mathrm{b}}$, Genevie `ve Milon ${ }^{\text {a }}$ \\ ${ }^{a}$ Unité d’Immunophysiologie et Parasitisme Intracellulaire, Département de \\ Parasitologie et Mycologie, \\ Institut Pasteur 25-28 rue du Dr Roux, 75724 Paris cédex 15, France \\ b Unité de Biologie des Interactions Hôte-Parasite, Département de Parasitologie et \\ Mycologie, \\ Institut Pasteur 25-28 rue du Dr Roux, 75724 Paris cédex 15, France \\ Received 23 January 2007; accepted 10 June 2007
}

\begin{abstract}
Control of human leishmaniases relies on appropriate diagnosis and reliable methods for monitoring chemotherapy. The current method used for estimation of parasite burden during chemotherapy patient follow-up as well as in pharmacological studies performed in experimental models involves PCR-based assays. Compared to time-consuming conventional methods, this type of Leishmania DNA detection-based method is extremely sensitive, but could fail in distinguishing viable Leishmania from slowly degenerating ones. We have used an in vitro model to monitor the duration of Leishmania DNA persistence in mouse macrophages following exposure to L-leucine ester, a molecule otherwise known to rapidly kill intracellular Leishmania amazonensis amastigotes. At one hour post L-leucine ester exposure, more than 98\% of amastigote-loaded macrophages harbored killed parasites and parasite remnants, as assessed by microscopy. This dramatic decrease in parasite load and the microscopic parasite follow-up over the $120 \mathrm{~h}$ time period studied correlated with Leishmania DNA as quantified by real-time PCR. Our results indicate that kinetoplast and nuclear parasite DNA degradation occurs very rapidly after amastigote death. These data add further weight to the argument that PCR assays represent not only a robust method for diagnosis but can also be reliable for monitoring parasite size reduction rate post any intervention (Leishmaniatargeting molecules, immunomodulators...).
\end{abstract}




\section{Introduction}

Leishmaniases are parasitic diseases that are endemic to nearly 90 countries, affecting some 12 million individuals in tropical, subtropical and Mediterranean regions [1]. The causal agents, Leishmania spp, are digenetic protozoan parasites belonging to the Trypanosomatidae family. The life cycle of these parasites requires two hosts, blood-feeding phlebotomine insects and mammals, including mainly rodents and carnivorous mammals. During their blood meal, Leishmania-housing vectors deliver the metacyclic infective promastigote stage into vertebrate host skin. Parasites are rapidly captured, mainly by macrophages, and then differentiate into their obligatory intracellular amastigote stage. Various clinical manifestations are described, ranging from less severe cutaneous forms to a fatal, if untreated, visceral form, depending on the parasite species and immunological status of the host [2]. Furthermore, a hallmark feature of the Leishmania parasite is the ability to reside in some mammalian hosts without inducing clinical symptoms [3, 4]. This asymptomatic parasitism assesses a dynamic equilibrium between distinct immune cells and molecules that either clear parasites or facilitate their persistence [5]. Disruption of this equilibrium in asymptomatic hosts can induce Leishmania multiplication and allow for the development of clinical symptoms [6].

To date, chemotherapy is the only intervention used for patients displaying clinical signs of the various forms of these parasitic diseases, since there are no effective preventive or therapeutic vaccines available [7]. Though the advent of effective new orally administered drugs such as Miltefosine, an alkylphosphocholine used primarily as an anti-cancer agent, represents a real hope for patients housing antimonial resistant Leishmania [8], chemotherapy of visceral leishmaniasis is far from satisfactory. In drug-treated patients, particularly those who are immunocompromised, it is of utmost importance to determine whether clearance of parasites has been achieved to avoid recurrence / relapses and complications. Methods based on parasite DNA detection, such as PCR, have proved highly specific and sensitive for monitoring parasite presence either for diagnosis or during follow-up of parasite load reduction in patients undergoing chemotherapy. Recently, some authors have evaluated the usefulness of PCR methods in assessing parasitological cure: a negative result was correlated with parasitological cure and a positive result with the presence of living parasites [9-11].

In order to establish the reliability of PCR for therapy monitoring in either drug-treated patients or animal models, it is important to know whether the parasite DNA detected and quantified by PCR exclusively assesses the presence of living amastigotes. In this study, we used a real-time PCR assay along with immunofluorescence and phase contrast microscopy to follow the parasite load fluctuations in Leishmania-harbouring macrophages that have been exposed to a leishmanicidal drug that acts very rapidly on amastigotes [12].

\section{Materials and methods}

\subsection{Mice and parasites}

Female Swiss nu/nu and BALB/c mice, between 8- and 12-weeks old, were obtained from Charles River (St Germain-sur l'Arbresle, France) and Janvier (Le Genest-St-Isle, France). L. amazonensis strain LV79 (MPRO/BR/1972/M1841) was propagated in Swiss nu/nu mice. Amastigotes were isolated from lesions and purified as described [13]. Promastigotes that had been differentiated from lesion-derived amastigotes were grown at $26^{\circ} \mathrm{C}$ in HOSMEM-II [13] supplemented with $20 \%$ heat-inactivated fetal calf serum (FCS, Dutscher, Brumath, France), $50 \mu \mathrm{g} / \mathrm{mL}$ of streptomycin and $50 \mathrm{IU} / \mathrm{mL}$ of penicillin (Antibiotics, Seromed, Berlin, Germany). Parasites were collected from Log phase cultures. 


\subsection{Preparation of macrophages and loading with L. amazonensis amastigotes}

Bone marrow cell suspensions recovered from tibias and femurs of BALB/c mice were suspended in RPMI 1640 medium (Seromed) supplemented with 10\% FCS, antibiotics (culture medium) and 20\% L-929 fibroblast-conditioned medium. Cells were distributed in bacteriologic Petri dishes (Greiner, Germany) and incubated at $37{ }^{\circ} \mathrm{C}$ in a $5 \% \mathrm{CO}_{2}$ air atmosphere. Six days later, bone marrow-derived smoothly adherent macrophages (BMDM) were washed with Dulbecco's phosphate buffered solution (PBS) and resuspended in prewarmed 1\% EDTA in Dulbecco's PBS without $\mathrm{Ca}^{+}$and $\mathrm{Mg}^{+}$(Biochrom $\mathrm{AG}$, Berlin, Germany). Recovered BMDM were deposited in flat-bottom 24-well plates (Tanner, Switzerland) at a density of $5 \times 10^{5}$ cells per well. For light microscopic studies, BMDM were allowed to adhere on $12 \mathrm{~mm}$ round glass coverslips. BMDM were then maintained in the same conditions as above, except that culture medium was supplemented with 4\% L-929 fibroblast-conditioned medium. Twenty-four hours after replating, BMDM nuclei were counted in control wells run in parallel [13] and amastigotes were added at a multiplicity of six amastigotes per host cell. BMDM cultures were incubated at $34{ }^{\circ} \mathrm{C}$, which is the permissive temperature for survival and multiplication of strain LV79 [14].

\subsection{Leishmanicidal treatment and parasite load follow-up}

The protocol used to kill intracellular amastigotes was adapted from Rabinovitch et al. [12]. Briefly, 24 hours after contact with parasites, BMDM cultures were incubated at $37{ }^{\circ} \mathrm{C}$ with RPMI-HEPES $20 \mathrm{mM}$, pH 7.4 supplemented with 3\% FCS, antibiotics and $2 \mathrm{mM} \mathrm{L-}$ Leucine methyl ester (Leu-oMe). After 1 hour of contact, Leu-oMe-containing medium was removed and replaced with either lysis medium for immediate DNA extraction and purification (see $\S 2.5$ ) or culture medium. In the latter case, all drug exposed and unexposed BMDM cultures were maintained at either $34{ }^{\circ} \mathrm{C}$ (any living intracellular amastigote develops) or at $37^{\circ} \mathrm{C}$ or $37.5^{\circ} \mathrm{C}$ (intracellular parasites die, 14). At various time points after Leu-oMe exposure (1 to $120 \mathrm{~h}$ ), BMDM cultures were processed for DNA isolation and fluorescence microscopy. All experimental points have been performed in duplicate.

\subsection{Microscopic analysis}

After fixation with paraformaldehyde, BMDMs were permeabilized and labeled with primary antibodies (Abs) and relevant fluorescent conjugates according to standard procedures [13]. Parasites were detected with parasite-specific mAb 2A3-26 [13]. Host cell molecules were detected with i) mAb specific for $\alpha$-tubulin (Clone DM 1A, Sigma-Aldrich, Milwaukee, WI, USA), and ii) biotinylated mAb specific for mouse Major Histocompatibility Complex class I molecules (clone 34-1-2S, eBioscience, San Siego, CA, USA). After immunolabeling, cell preparations were incubated for 5 min with $5 \mu \mathrm{g} / \mathrm{ml}$ Hoechst 33342 (Molecular probes, Invitrogen SARL, Cergy Pontoise, France) in PBS to stain the nuclei of host cells and parasites. Coverslips were mounted in Mowiol (Calbiochem, San Diego, CA) before observation under an inverted microscope Zeiss Axiovert 200M piloted by Zeiss Axiovision 4.4 software (Carl Zeiss Microscopy, Jena, Germany). Images were acquired with a black and white CCD camera (Roper Scientific GmbH Germany) and further processed using Photoshop software (Adobe ${ }^{\circledR P h o t o s h o p}{ }^{\circledR C S}$ ). 
Leishmanicidal effects were monitored by two parameters: (a) the reduction of parasitophorous vacuole (PV) size and number and (b) the overall decrease in intracellular amastigote number.

\subsection{Cell lysis and DNA isolation}

Cell lysis and DNA isolation from adherent BMDMs, cultured promastigotes and lesionderived purified amastigotes, were performed with the DNeasy Tissue kit (Qiagen, Courtaboeuf, France), according to the manufacturer's instructions.

\subsection{Leishmania and mouse macrophage DNA targets selected for amplification}

Sets of primers generating Leishmania genomic or kinetoplastic DNA amplification products were designed using unique or multiple sequences retrieved from NCBI databanks (http://www.ncbi.nlm.nih.gov/). Alignment of sequences was generated with the MultAlin algorithm [15, http://prodes.toulouse.inra.fr/multalin/multalin.html]. Oligonucleotide pairs were chosen using the LightCycler Probe Design software (version 1.0, Idaho Technology Inc.). Similarities of primer sequences with those present in nucleotide databanks were validated using the BLAST program (http://www.ncbi.nlm.nih.gov/BLAST/).

The mouse-specific housekeeping gene beta-catenin ( $\beta$-cat) was amplified to monitor i) sample DNA degradation, ii) presence of potential inhibitors of PCR or iii) variation in DNA yield. Characteristics of targets and primers used in this study are summarized in Table 1.

\subsection{Real-time PCR conditions and cycling parameters}

PCR was performed using a LightCycler ${ }^{\circledR} 480$ system (Roche Diagnostics, Meylan, France) and set up in white opaque polypropylene wells (LightCycler ${ }^{\circledR} 480$ Multiwell Plates 384) in a final volume of $10 \mu \mathrm{l}$ per reaction. Briefly, $1 \mu \mathrm{l}$ of sample DNA was added to $9 \mu \mathrm{l}$ of a master mix containing $5 \mu \mathrm{l}$ of QuantiTect SYBR Green Kit (Qiagen) and $4 \mu \mathrm{l}$ of nucleasefree water with primers (Guaranteed Oligos ${ }^{\mathrm{TM}}$, Sigma-Aldrich) at a final concentration of 0.5 $\mu \mathrm{M}$. Activation of the Qiagen Thermophilus aquaticus polymerase was performed at $95{ }^{\circ} \mathrm{C}$ for $15 \mathrm{~min}$. The PCR program included 40 cycles of denaturation at $95^{\circ} \mathrm{C}$ for $10 \mathrm{~s}$, annealing at $54{ }^{\circ} \mathrm{C}$ for $25 \mathrm{~s}$ and extension at $72{ }^{\circ} \mathrm{C}$ for $30 \mathrm{~s}$. SYBR Green fluorescent emission was measured at the end of the elongation step. Subsequently, a melting curve program was applied with a continuous fluorescent measurement starting at $70{ }^{\circ} \mathrm{C}$ and ending at $95{ }^{\circ} \mathrm{C}$ (ramping rate of $0.1^{\circ} \mathrm{C} / \mathrm{s}$ ).

\subsection{Specificity and sensitivity of the PCR assay}

Product identification was performed using a melting curve analysis at the end of each PCR. Melting curves were converted to melting peaks and the Tm for each amplicon was determined (Table 1). PCR products were checked after migration in a $1.5 \%$ agarose gel in TBE stained with ethidium bromide.

Sensitivity assays included BMDM cultures spiked with defined numbers of amastigotes immediately prior to the lysis process and 10-fold serial dilutions of DNA from lesion-derived purified amastigotes or log-phase promastigotes. 


\subsection{Analysis of PCR data}

Mean Crossing Point values (Cp) were determined by the second derivative maximum method of the LightCycler ${ }^{\circledR} 480$ Basic Software. Cp were used for relative quantitative data analysis using the Pfaffl method [16]. The relative expression ratio $\mathrm{R}$ of a parasite gene was computed based on the amplification efficiency (E) and Cp deviation between a sample $\left(\mathrm{Cp}_{\text {sample }}\right)$ and a control (L. amazonensis-loaded BMDM before Leu-oMe exposure; $\mathrm{Cp}_{L a-}$ вмDм). Cp values for the host $\beta$-cat reference gene were used for data normalization.

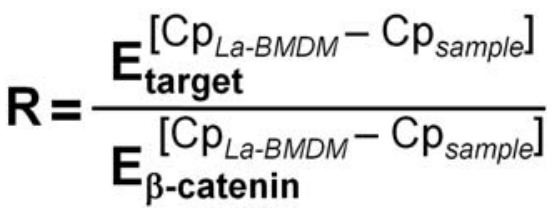

Amplification efficiency of each target was determined according to the equation $\mathrm{E}=10^{(-}$ $1 / S)$, where $S$ is the slope of the standard curve generated from ten-fold serial dilutions of purified parasite or host cell DNA.

Data processing and presentation were performed using Microsoft Excel and SigmaPlot 2004 software (Systat), respectively.

\section{Results}

The aim of the present study was to determine the outcome of Leishmania DNA post exposure of intracellular amastigotes to a rapidly acting leishmanicidal molecule. The leishmanicidal amino acid ester Leu-oMe induces massive, immediate killing of intracellular amastigotes of the L. mexicana complex [12]. The killing mechanism involves lysosomal compartments (megasomes) found only within members of this Leishmania complex, and relies on their acidic hydrolase activities [17]. Application of this simple and swift protocol allowed for efficient monitoring of $L$. amazonensis amastigote outcome as well as parasite DNA fate. We also benefited from the temperature sensitivity of strain LV79, which stops growing and eventually dies when incubated at $37{ }^{\circ} \mathrm{C}$ (this study) or $37.5^{\circ} \mathrm{C}$ [14] for a prolonged period of time.

\subsection{Microscopic follow-up of parasite load in Leu-oMe-exposed L. amazonensis-harboring BMDMs}

Twenty-four or forty-eight hours after onset of intracellular parasitism, L. amazonensisloaded BMDMs showed a regular shape and usually contained one or more large PVs housing amastigotes (Fig. 1A, 1B: white stars). Numerous parasites were seen as smooth, rice shaped bodies, usually attached to the PV membrane (Fig. 1A, 1B: white arrows). Hoechst staining of nucleic acids allowed for visualization of nuclei from both host macrophages and amastigotes (Fig. 1: blue). The presence of intracellular parasites with intact morphology (Fig. 1A, 1B) correlated with the detection of their nuclei (Fig .1B, 1C).

After one hour of exposure to Leu-oMe at $37^{\circ} \mathrm{C}$, there was a sharp decrease in parasite burden as evidenced by the low percentage $(<1 \%)$ of BMDMs still harboring amastigotes with normal morphology and attached to the PV membrane, compared to $>98 \%$ in cultures at $34^{\circ} \mathrm{C}$ not exposed to Leu-oMe (Fig. 1 and data not shown). In most microscopic fields observed, neither morphologically intact amastigotes nor parasite nuclei could be evidenced (Fig. 1E, 1F). However, parasite remnants were still detected by fluorescence (Fig. 1D, green, cyan arrows) and differential interference contrast microscopy (Fig. 1E, cyan arrows). For cell cultures maintained at $37^{\circ} \mathrm{C}$ following Leu-oMe exposure, the percentage of L. amazonensis- 
loaded BMDMs remained stable from 5 to $24 \mathrm{~h}$ and decreased after $48 \mathrm{~h}$ to almost undetectable levels at $120 \mathrm{~h}$ (data not shown).

At $34{ }^{\circ} \mathrm{C}$ and until $48 \mathrm{~h}$ post Leu-oMe exposure, only minute numbers of unaltered intracellular amastigotes could be counted in the PVs of rare BMDMs containing parasites (Fig. 2D-F, white arrows) compared to Leu-oMe-unexposed BMDMs, which harbored more than 10 parasites per cell (Fig. 2A-C).

\subsection{Leishmania DNA targets and selection of primers for real-time PCR assays}

We have designed primers for various Leishmania target genes to follow the fate of Leishmania DNA: mitochondrial or kinetoplast DNA (kDNA) and nuclear DNA. Sequences for DNA polymerase $\alpha$ (DNA Pol $\alpha$ ), glucose 6-phospate dehydrogenase (G6PD), hexokinase and the small subunit ribosomal RNA (ssr RNA, multy-copy gene) were retrieved from the NCBI database. Primers were designed and selected according to the guidelines described in $\S$ 2.6 Primers obtained following this procedure could thus be used in one unique PCR protocol as defined in §2.7. Modified published primer sequences were used to detect kDNA minicircle targets (JW11 and JW12, [9, 18]). Primers specific for host $\beta$-cat DNA gene were designed to compensate for inter-PCR variations and differences in DNA yields between samples, As an initial control, the PCR product of each target was analyzed on an agarose gel to confirm the single product size (data not shown) and, subsequently, the Tm was determined to assess the specificity of the amplicons (Table 1). Absence of primer cross-reactivity between host cell and Leishmania DNA was assessed with various template amounts from both organisms (data not shown). All relevant information about the primers and amplicons is displayed in Table 1.

The dynamic range of the real-time quantitative Leishmania PCR test was determined by serial dilutions of $L$. amazonensis DNA in water. Representative data for all Leishmania targets are shown in Figure 3. Amplicon detection was linear over a 6 log-fold range of Leishmania DNA concentrations and the assay allowed us to accurately detect as few as 100 fg of parasite DNA (Fig. 3A). PCR efficiency for each target was determined from the $E=10^{[-}$ 1/slope] equation, plotting the $\mathrm{Cp}$ values against the log of the initial template concentration (Fig. 3B). Efficiencies were found to be high and constant with a strong correlation coefficient $\left(\mathrm{R}^{2}>0.99\right)$ over the concentration/dilution range studied (Fig. 3B and data not shown). ssrRNA- and kDNA-specific primers showed higher sensitivity, as repeatedly demonstrated by lower Cp values compared to other nuclear DNA targets (data not shown). However, after 38 PCR cycles, the increase in fluorescence values detected was deemed unreliable due to Poisson distribution statistics. Positive detection from approximately $10 \mathrm{fg}$ of parasite DNA suggested nearly single-parasite sensitivity.

Only one melting peak with a near constant value was observed for each PCR amplification product (Table 1). For example, the Tm of ssrRNA amplicons calculated from melting curves was $79.07^{\circ} \mathrm{C}+/-0.08^{\circ} \mathrm{C}$ (Fig. 3C). No primer-dimers were detected except for the kinetoplastic minicircle target, the detection of which was based on the use of JW11/JW12 primers (Fig. 3C, Table 1 and data not shown).

\subsection{PCR assays on BMDMs harboring L. amazonensis amastigotes}

Efficacy of the combined procedure of PCR (DNA extraction and purification steps) was first verified. To this end, BMDM samples spiked with defined amounts of Leishmania amastigotes were prepared. Highly reproducible results were obtained for each spiked control (performed in duplicate samples) and high linearity was maintained over the range of template parasite numbers added (Fig. 4 and data not shown). Presence of host cell DNA did 
not hinder the detection of Leishmania DNA, even in the presence of very few parasites i.e. 0.2 parasites per PCR (10 parasites per well).

\subsection{Parasite DNA is rapidly degraded once the parasite is killed}

PCR results for the detection of parasite DNA are expressed as fold differences (termed R) in L. amazonensis gene copies relative to mouse host gene copies (see §2.9 of Materials and Methods). After $1 \mathrm{~h}$ of Leu-oMe exposure, less than $1 \%$ of the initial leishmanial nuclear DNA content could be detected by PCR using ssrRNA specific primers (Fig. 5). Under these conditions, the leishmanicidal activity of Leu-oMe resulted in swift and almost complete killing of intracellular amastigotes. When the culture was subsequently maintained at $37^{\circ} \mathrm{C}$, the parasite burden slowly decreased to very low levels at $120 \mathrm{~h}$ post Leu-oMe exposure. The same parasite DNA detection profile pattern was obtained using primers specific for other nuclear genes studied (DNA Pol, Hexokinase and G6PD) as well as with primers that amplify conserved regions of kinetoplast minicircles (data not shown).

A few hours after Leu-oMe exposure, the low parasite burden determined by PCR was correlated with detection of very small numbers of unaltered intracellular amastigotes by microscopy. Furthermore, in parallel experiments and until $48 \mathrm{~h}$ post leishmanicidal treatment, positive cultures for promastigote growth were obtained from BMDMs maintained at $26{ }^{\circ} \mathrm{C}$ in HOSMEM-II medium (data not shown). These data revealed the presence of living amastigotes that have resisted the single shot leishmanicidal effect of Leu-oMe and were able to differentiate into promastigotes and multiply in the relevant medium. When the cell culture was maintained at $34{ }^{\circ} \mathrm{C}$, the few living parasites detected at $5 \mathrm{~h}$ could resume their growth as demonstrated by the progressive increase in relative expression values calculated at 24, 48 and $120 \mathrm{~h}$ (Fig. 5). Moreover, the temperature shift from $34{ }^{\circ} \mathrm{C}$ to $37{ }^{\circ} \mathrm{C}$ without drug treatment had a progressive leishmanicidal effect which became detectable after $48 \mathrm{~h}$. Very few parasite-harbouring BMDMs could be detected after $120 \mathrm{~h}$ in cultures that had been exposed to leishmanicidal treatment (Fig. 5). In control experiments performed at $34{ }^{\circ} \mathrm{C}$, the amount of Leishmania DNA in BMDMs left unexposed to Leu-oMe slowly increased over the time period studied.

\section{Discussion}

Conventional methods used for leishmaniasis diagnosis can also be used to assess therapeutic regimens for efficiency of Leishmania clearance. Indeed, limiting dilution assays [19] allow for the quantification of parasites present within tissue biopsies but, although sensitive, these culture-based methods are tedious and time-consuming. Moreover, not all parasites are cultivable. The ex vivo / in vitro growth and subsequent quantification of Leishmania promastigotes by limiting dilution assays may be impeded by the Leishmania species used, the composition of the medium (FCS and other additives) and / or the presence of host tissue material. This is particularly evident when parasites are scarce [3, 4, 20 and unpublished observations]. Moreover, many current serological methods cannot distinguish between current, past or asymptomatic Leishmania-driven parasitism. Due to its high sensitivity and specificity, the use of real-time PCR may offer a number of advantages over conventional assays to monitor parasite load outcome in human patients undergoing chemotherapy or in pharmacological studies developed in experimental models.

However, the question of whether Leishmania DNA detected and quantified by PCR is indicative of live parasites represents a critical issue that needs to be addressed. This study was designed to investigate the fate of Leishmania DNA within BMDMs that were loaded with $L$. amazonensis amastigotes and briefly exposed to a leishmanicidal molecule. We 
adapted a protocol based on the use of Leu-oMe, an experimental leishmanicidal drug that only acts on intracellular amastigotes belonging to $L$. amazonensis or $L$. mexicana species. This drug, which induces rapid death, was used to avoid the problem of detecting DNA originating from a mixed population of living and dead organisms at each given time point. Indeed, commercially available drugs are known to act progressively over a long period of time. After one hour of exposure to this amino acid ester, we noticed a decrease in parasite burden of more than 98\%, as assessed by both microscopy and real-time PCR. Validation of the real-time PCR method by another established method i.e. the identification of unaltered amastigotes by microscopy, allowed for a high correlation between PCR values and living parasite numbers to be established [this study and 21]. Furthermore, the survival of some amastigotes post-leishmanicidal treatment was evidenced in parallel experiments by their ability to differentiate into promastigotes and multiply in culture (data not shown). Exposing BMDM cultures to $34{ }^{\circ} \mathrm{C}$ after Leu-oMe exposure allowed for the detection of a progressive increase in parasite load by real-time PCR. This observation highlights the fact that intracellular surviving $L$. amazonensis amastigotes were able to resume growth at a permissive temperature. Thus, our results demonstrated that Leishmania DNA detected by PCR is derived from intact parasites and that nuclear DNA and kDNA are rapidly degraded following amastigote death. These data are in agreement with those from recent clinical studies, in which a positive PCR result was correlated to the presence of living parasites while a negative result was obtained after parasitological cure [10,11, 22]. This concordance is not restricted to Leishmania and to leishmaniases. Indeed, studies on other eukaryotic protozoan parasites such as Plasmodium chabaudi, Trypanosoma cruzi, and Toxoplasma gondii also strengthen the observation that a positive PCR result assesses the presence of viable parasites [23 - 25]. Interestingly, this is in contrast to assessment of bacterial DNA, especially Mycobacterium [26 - 30].

In most clinical studies in which PCR has been used to monitor the presence of Leishmania, parasite DNA remained detectable in all cured individuals who experienced relapse. However, some cured individuals who were PCR negative could still develop clinical symptoms, while others exhibiting Leishmania-DNA positive PCR did not develop disease [22]. This indicates that attention should be paid to the sensitivity threshold of the PCR assay used as well as the necessity to perform a kinetic detection study, not just end-point detection. In our study, the detection limit was around 0.2 to 1 parasites for $1 \mu \mathrm{l}$ of sample. In other studies, in which optimization of extraction methods and PCR conditions were enhanced (end point or real-time PCR), the sensitivity threshold fell below 0.01 parasites per PCR, which should allow for accurate follow-up of parasite load in patients benefiting from therapeutic interventions [21, 29, 30].

In conclusion, our data add further weight to the argument that the PCR assay represents not only a robust method of diagnosis, but can also be a reliable tool for monitoring the efficacy of Leishmania-targeting molecules as well as an early, preclinical, prognostic marker of relapse.

Acknowledgments:

We apologize to authors for failing to cite their work directly owing to space constraints.

The authors are mostly indebted to Roche Diagnostics (Meylan, France) for providing them with the LightCycler ${ }^{\circledR} 480$ system.

This work was supported by Le Fonds Dédié numéro 13, Combattre les Maladies Parasitaires (Sanofi-Aventis, Institut Pasteur and Ministère de la Recherche).

The authors have no conflicting financial interests. 
Figure 1. Microscopic follow-up of intracellular L. amazonensis amastigotes in BALB/C BMDMs $1 \mathrm{~h}$ post exposure to Leu-oMe.

Leishmania-harboring macrophages were grown at $34^{\circ} \mathrm{C}(\mathrm{A}, \mathrm{B}, \mathrm{C})$ or exposed at $37^{\circ} \mathrm{C}$ to LeuoMe for $1 \mathrm{~h}$ (D, E, F) before fixation and processing for immunofluorescence microscopy analysis. Intracellular amastigotes were stained with Alexa-488-conjugated specific mAb 2A3.26 (A, D). Host intracellular (A) or membrane (D) macrophage molecules were stained using anti $\alpha$-tubulin and anti MHC Class I molecule Ab, respectively, followed by Texas Red conjugated secondary Abs. Host macrophage and parasite DNA were stained with Hoechst dye. Black and white images acquired with the CCD camera were colorized according to standards used in fluorescence microscopy: green: Alexa-488, red: Texas Red and blue: Hoechst.

The presence of intact parasites and the lumen of PVs are indicated by white arrows (A, B) and white asterisks (A, B, C), respectively. Following Leu-oMe exposure, only parasite remnants could be detected (cyan arrows, D, E). Note the absence of DNA staining for drugexposed Leishmania amastigotes (E, F) compared to healthy parasites (B, C). A net correlation is observed between DNA detection and parasite morphology as revealed by differential interference contrast microscopy (B, E).

A yellow color indicates co-localisation or very tight association of the molecules concerned (A, D). Anti $\alpha$-tubulin mAb also recognizes Leishmania tubulin (data not shown).

Figure 2. Microscopic follow-up of intracellular L. amazonensis amastigotes in BALB/C BMDMs $48 \mathrm{~h}$ post exposure to Leu-oMe.

Leishmania-harboring macrophages were left unexposed at $34^{\circ} \mathrm{C}$ (A, B, C) or exposed at $37^{\circ} \mathrm{C}$ to Leu-oMe for $1 \mathrm{~h}$ and then left unexposed at $34^{\circ} \mathrm{C}$ for $48 \mathrm{~h}(\mathrm{D}, \mathrm{E}, \mathrm{F})$ before fixation and processing for immunofluorescence microscopy analysis. Staining of parasites, $a$-tubulin, MHC class I molecules and nucleic acids along with images' colorization were performed as described in the legend to Fig.1.

In macrophages unexposed to Leu-oMe, numerous parasites are detected within large PVs (green-yellow staining, A; smooth rice-shaped bodies, B). Each amastigote is associated with DNA staining (blue spot, B, C).

After Leu-oMe exposure and following a $48 \mathrm{~h}$ incubation period at $34^{\circ} \mathrm{C}$, the permissive temperature for LV79 strain development, very few intact parasites could be visualized by immunofluorescence or differential interference contrast microscopy (D, E, F; white arrows). Note that nucleic acid staining is only associated with intact amastigotes (E, F, white arrows). In cured macrophages, PVs have been reduced or have disappeared completely but parasite remnants are still detected (green-yellow staining, D), sometimes associated with granular structures revealed by differential interference contrast microscopy (E) but without DNA costaining (E, F).

Figure 3. Sensitivity and specificity of the real-time PCR assay.

(A) Amplification plots for the ssrRNA gene from serial 10-fold dilutions of L. amazonensis DNA (B) Plot of mean Cp values +/- 1 S.D. (technical duplicates) against the logarithmic quantity of Leishmania DNA (10 ng to $100 \mathrm{fg}$ per reaction). Cp values were extracted from graph (A) fluorescence values using the second derivative maximum method. (C) Melting peaks were determined from the first negative derivative of the fluorescence versus the temperature i.e. melting curves for each amplicon.

Figure 4. Reproducibility and sensitivity of PCR assays from spiked controls.

BMDMs were seeded with increased numbers of purified amastigotes before total DNA isolation. Relative quantity (R) represents the normalized ratio of Leishmania (L) DNA target 
quantity (ssrRNA) over Mus (M) $\beta$-cat DNA. The calibrator for comparison between samples was Leishmania-loaded BMDMs before Leu-oMe exposure $(\mathrm{R}=1)$. Sensitivity for ssrRNA was approximately 0.2 parasite per PCR for 10 parasites added per well.

Figure 5. Real-time PCR follow-up of parasite DNA after exposure to Leu-oMe.

At different time points post Leu-oMe addition to LV79 amastigote-loaded BMDMs, total DNA was isolated and real-time PCR assays were performed to quantify Leishmania (L) DNA targets. As a representative example, the relative quantity (R) of ssrRNA target was expressed comparatively to the Mus (M) $\beta$-cat DNA for each sample. Data were expressed using the sample Leishmania-loaded BMDM before Leu-oMe addition as a calibrator $(\mathrm{R}=1)$. Leu-oMe-exposed macrophages are represented by squares and those not exposed to drug by circles. Macrophage cultures were maintained either at $37^{\circ} \mathrm{C}$ (red color) or $34^{\circ} \mathrm{C}$ (blue color).

These data are representative of three experiments. 